Check for updates

Cite this: RSC Adv., 2017, 7, 43909

\title{
An asymmetric wettable chitosan-silk fibroin composite dressing with fixed silver nanoparticles for infected wound repair: in vitro and in vivo evaluation $\dagger$
}

Jinglong Liu, to ${ }^{\text {ab }}$ Zhiyong Qian, (ID +t ${ }^{\text {cd }}$ Quan Shi, ${ }^{a}$ Shuo Yang, ${ }^{a}$ Qianxin Wang, ${ }^{a}$ Bo Liu, ${ }^{a}$ Juan Xu, ${ }^{* a}$ Ximin Guo*d and Haifeng Liu (D) *c

The treatment of large-area infected wounds remains a significant challenge, as there is no effective wound dressing for infected wound healing applicable to clinical applications. In this study, chitosan-silk fibroin composite scaffolds containing silver nanoparticles (AgNP) that underwent asymmetric modification were fabricated for tissue engineering dressings. The scaffolds were prepared from a solution containing a chitosan-silk-fibroin emulsion with added AgNPs using a lyophilization approach and with an asymmetric coating on the surface of one side. Transmission electron microscopy images and laser particle size analysis showed the AgNP distribution, scanning electron microscopy images showed the surface morphology of the dressings, and inductively coupled plasma mass spectrometry showed the silver content. The asymmetric wettability of the dressings was measured using a contact angle meter. High porosity, good moisture retention capability and appropriate tensile strength were observed by measuring the physical and mechanical properties. Good antimicrobial properties towards various bacteria were observed via in vitro antibacterial effect analysis. Controlled AgNP release by the dressings and their resistance to the infiltration of microorganisms were also observed in our experiments. A highly biocompatible dressing was observed by the MTT assay experiment and subcutaneous sensitization test. Moreover, the effects of the dressings on infected wound healing were measured in mice infected wound models. Analysis of the wound healing rate, bacterial cultures of the exudate, blood samples and histological examination all provided satisfactory results. These results demonstrate that the dressings we prepared offer the potential for infected wound tissue repair and regeneration.

Received 10th July 2017

Accepted 29th August 2017

DOI: $10.1039 / c 7 r a 07588 j$

rsc.li/rsc-advances

\section{Introduction}

As the largest organ in humans, the skin not only maintains our metabolism by preventing evaporation but also plays an important role in protection against UV irradiation, chemical substances, foreign organisms and other harmful environmental factors. ${ }^{1-3}$ However, trauma and burns often lead to tissue necrosis and disrupt the defence functions of skin.

${ }^{a}$ Department of Stomatology, The Chinese PLA General Hospital, Beijing, 100853, P. R. China.E-mail:drxujuan@126.com

${ }^{b}$ Department of Stomatology, The General Hospital of the PLA Rocket Force, Beijing, 100088, P. R. China

${ }^{c}$ School of Biological Science and Medical Engineering, Beihang University, Beijing, 100083, P. R. China. E-mail: haifengliu@buaa.edu.cn

${ }^{d}$ Department of Advanced Interdisciplinary Studies, Institute of Basic Medical Sciences, People's Liberation Army Military Medical Science Academy of the PLA, Beijing, 100850, P. R. China.E-mail: guoxim@163.com

$\dagger$ Electronic supplementary information (ESI) available. See DOI: 10.1039/c7ra07588j

\$ Jinglong Liu and Zhiyong Qian are co-first authors.
Wound infection is the most common complication of skin damage and is a major cause of delayed healing., ${ }^{\mathbf{4} 5}$ Severe wound infection is associated with the occurrence of bacteremia and sepsis and may even lead to the development of systemic inflammatory response syndrome (SIRS) and multiple organ dysfunction syndrome (MODS). ${ }^{6-8}$ Topical drugs are widely used for the treatment of burn wounds. However, when these drugs are used to treat large-area skin wounds, large doses and high administration frequencies are typically required, which often cause varying degrees of side effects. ${ }^{9-14}$ Therefore, improving wound dressings is of great importance for the effective treatment of infected wounds. An ideal wound dressing should retain moisture, maintain electrolyte balance and haemostasis, provide analgesic and antibacterial properties, and promote wound healing. ${ }^{15,16}$ The two sides of a dressing should be equipped with different characteristics. The inside surface (i.e., the side next to the skin) should have good hydrophilicity, enable the dressing to attach well to the wound, and play a superior role in regenerating skin tissue and fighting infection. The outside of the dressing (the side exposed 
to the air) should exhibit hydrophobicity, which can enable the dressing to act as a barrier against outside risk factors. ${ }^{17-19}$ Although some wound dressings, such as Syvek-Patch, Chitopack C, Tegasorb, HemCon Bandage, and KytoCel, have been applied in clinical practice and are commercially available, ${ }^{15,20-23}$ their treatment outcomes are far from satisfactory. As a type of artificial antibacterial material, silver nanoparticles (AgNP) have a small particle size, large surface area, and broadspectrum bactericidal effects without causing resistant bacteria. Over the last few decades, the preparation of antibacterial dressings by combining AgNP with polymers has become an important area of research. For example, $\mathrm{Mandal}^{24}$ and Duraipandy ${ }^{25}$ prepared antibacterial dressings by combining AgNP and collagen. However, these researchers did not dissymmetrically modify their dressings, and thus, the moisture retention of the dressings was relatively poor. Additionally, collagen is derived from heterogeneous animals and may trigger an immune response when it is not specially processed. For this reason, the application of collagen in clinical practice is restricted. Liang ${ }^{26}$ used a chitosan sponge with superior biocompatibility to collagen to adsorb AgNP and asymmetrically modified the material to increase its moisturizing properties. However, the structural characteristics of chitosan made the dressing brittle, and the particles were not strongly adsorbed, which caused them to be easily lost. To resolve the above limitations of antibacterial dressings, a sponge scaffold containing AgNP was produced in this study by blending and emulsifying chitosan and silk fibroin, adding a specific concentration of a AgNP solution and then freeze drying the final solution. Chitosan and silk fibroin exhibit good tissue repair function. Silk fibroin fibre can control the release of AgNP and endow the dressing with good flexibility. Finally, an antibacterial dressing with an asymmetric coating was prepared by grafting stearic acid (SA) onto the smooth surface. We obtained an asymmetric wettable chitosan-silk fibroin composite dressing with fixed AgNP (CTS-SF Ag/SA). The CTS-SF Ag/SA dressing promotes tissue regeneration and exhibits antiinfection and moisture-retention properties. The silver content, physical properties, cytotoxicity and antibacterial capability of the dressing as well as its effectiveness on infected wounds were evaluated.

\section{Materials and methods}

\subsection{Materials}

Chitosan (degree of deacetylation $\geq 95 \%$ ) was obtained from Qingdao Haihui Biotechnology Co., Ltd., China. Bombyx mori silk was purchased from Yi Xian Raw Silk Factory in China. Anhydrous sodium carbonate and anhydrous calcium chloride were obtained from Beijing Seasky Bio Technology Co., Ltd. Glucose, polyvinylpyrrolidone (PVP), sodium hydroxide $(\mathrm{NaOH})$, and cetyltrimethyl ammonium bromide (CTAB) were obtained from Beijing Hengye Zhongyuan Chemicals Co., Ltd., China. Silver nitrate, stearic acid (SA), absolute ethyl alcohol, and dicyclohexylcarbodiimide (DCC) chloroform were obtained from Sinopharm Chemical Reagent Co., Ltd., China, Escherichia coli (ATCC25922), Staphylococcus aureus (ATCC25923),
Pseudomonas aeruginosa (ATCC27853) and Monilia albicans (ATCC64548) were obtained from the Chinese PLA General Hospital. DMEM medium (Gibco, USA) and foetal bovine serum (FBS) were obtained from MDgenics Inc., USA. L929 cells (American Type Culture Collection, Manassas, VA, USA) were supplied by our laboratory, human fibroblast cells (HFCs) were supplied by the Department of Burn and Plastic Surgery at the Chinese PLA General Hospital, and human umbilical cord mesenchymal stem cells (HUCMSCs) were supplied by the Department of Obstetrics and Gynecology at the Chinese PLA General Hospital. BALB/c mice were obtained from the Beijing Vital River Laboratory Animal Technology Co., Ltd., and guinea pigs were obtained from the Beijing Keyu Animal Aquaculture Centre.

\subsection{Preparation of the CTS-SF Ag/SA dressings}

2.2.1 Preparation of the CTS-SF and CTS-SF Ag dressings. Bombyx mori silk was submerged in a $0.5 \mathrm{wt} \%$ sodium carbonate solution for $30 \mathrm{~min}$ at $90-100{ }^{\circ} \mathrm{C}$ and then washed with distilled water to remove sericin. A $12 \%(\mathrm{w} / \mathrm{v})$ calcium dichloride/ethanol solution was prepared and dissolved in a ternary solvent $\left(\mathrm{CaCl}_{2}: \mathrm{CH}_{3} \mathrm{CH}_{2} \mathrm{OH}: \mathrm{H}_{2} \mathrm{O}=1: 2: 8\right)$ at $70{ }^{\circ} \mathrm{C}$, and after magnetic stirring for $1 \mathrm{~h}$, the mixed solution was obtained. The mixed solution was loaded in a dialysis bag and dialysed in a cellulose tube against distilled water for 3 days at room temperature to remove salts. After vacuum filtration, the silk fibroin solution was obtained.

First, $0.5 \mathrm{~g}$ silver nitrate was dissolved in $1 \mathrm{ml}$ distilled water to obtain the silver nitrate solution. Next, $1 \mathrm{~g}$ glucose, $1 \mathrm{~g}$ PVP, $0.2 \mathrm{~g} \mathrm{NaOH}$, and $1 \mathrm{~g}$ CTAB were added to $40 \mathrm{ml}$ distilled water and stirred until completely dissolved to obtain a mixed solution. The silver nitrate solution was added to the mixed solution, which was then mechanically agitated at $1500 \mathrm{rpm}$ at $60{ }^{\circ} \mathrm{C}$ for $2 \mathrm{~h}$. The particles were collected by ultracentrifugation (30 $000 \mathrm{rpm}$; $30 \mathrm{~min}$ ), redispersed in pure water and collected again by ultracentrifugation. Then, a $600 \mu \mathrm{g} \mathrm{ml}{ }^{-1}$ AgNP solution was prepared with distilled water, as shown in Fig. 1a and b.

Then, $10 \mathrm{ml}$ of a $2 \%$ of chitosan solution was added to $20 \mathrm{ml}$ of a $4 \%$ silk fibroin solution and mechanically agitated at $1500 \mathrm{rpm}$ for $30 \mathrm{~min}$. The chitosan-silk-fibroin emulsion was poured into a container (basal area: $200 \mathrm{~mm} \times 300 \mathrm{~mm}$ ) and allowed to sit at $-20{ }^{\circ} \mathrm{C}$ for $12 \mathrm{~h}$, followed by $-70{ }^{\circ} \mathrm{C}$ for $6 \mathrm{~h}$, and then freeze-dried using a vacuum freeze drier (FD-27s, Beijing DETIANYOU Technology Development Co., Ltd.), resulting in the CTS-SF dressing.

Next, $0.5 \mathrm{ml}, 1 \mathrm{ml}$, and $2 \mathrm{ml}$ of the AgNP solution were separately added to $30 \mathrm{ml}$ of a chitosan-silk fibroin solution, and each solution was poured into one of three containers (basal area: $200 \mathrm{~mm} \times 300 \mathrm{~mm}$ ). The mixtures were maintained at $-20{ }^{\circ} \mathrm{C}$ for $12 \mathrm{~h}$, followed by $-70{ }^{\circ} \mathrm{C}$ for $6 \mathrm{~h}$, and then freezedried, resulting in CTS-SF dressings with different AgNP concentrations (denoted CTS-SF Ag0.5, CTS-SF Ag1.0 and CTSSF Ag2.0, respectively) (Fig. 1 steps 1-4).

The dressings (CTS-SF, CTS-SF Ag0.5, CTS-SF Ag1.0 and CTSSF Ag2.0; basal area: $50 \mathrm{~mm} \times 50 \mathrm{~mm}$ ) were soaked in deionized water for at least $24 \mathrm{~h}$ and then frozen at $-20{ }^{\circ} \mathrm{C}$ for $12 \mathrm{~h}$. 


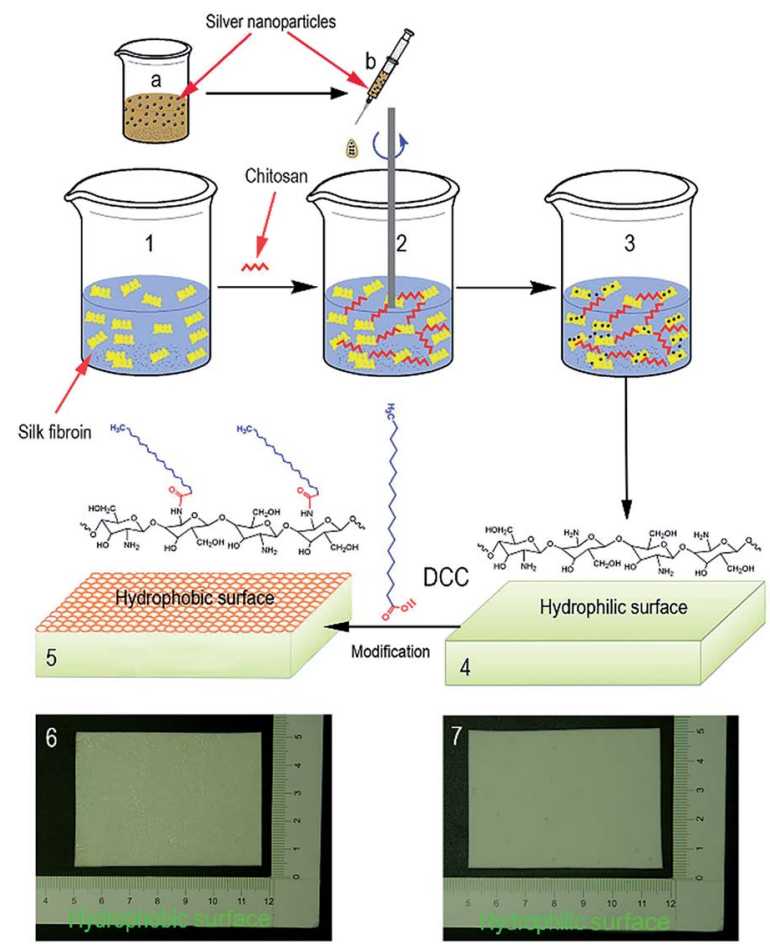

Fig. 1 Synthesis and schematic diagram of the CTS-SF Ag/SA dressing The CTS-SF Ag dressing was lyophilized for $24 \mathrm{~h}(1-4)$, and then the dressing was modified by stearic acid (5) finally, the dressing was prepared $(6,7)$.

After that, $1 \mathrm{ml}$ of stearic acid solution $\left(40 \mathrm{mmol}^{-1}\right.$ in alcohol, DCC as a dehydrating agent) was poured uniformly onto the smooth surface of each frozen dressing, and the dressings were kept frozen at $-20{ }^{\circ} \mathrm{C}$ for $1 \mathrm{~h}$. The smooth surface of each frozen dressing was rinsed 3 times with absolute alcohol at $-20{ }^{\circ} \mathrm{C}$. Subsequently, the frozen samples were lyophilized to obtain asymmetrically wettable dressings and labelled CTS-SF/SA, CTSSF Ag0.5/SA, CTS-SF Ag1.0/SA and CTS-SF Ag2.0/SA, respectively. The final dressings are shown in Fig. 1(6 and 7).

\subsection{Physical properties test}

2.3.1 Characterization. The AgNP were characterized using a transmission electron microscope (TEM, Philips Tecnai G220, accelerating voltage of $200 \mathrm{kV}$ ). The diameter of the AgNP was analysed by a laser particle size analyser (BT-9300S, Dandong BaiTe Science and Technology Co., Ltd.). The morphologies of the CTS-SF Ag0.5/SA, CTS-SF Ag1.0/SA and CTS-SF Ag2.0/SA dressings were examined under a scanning electron microscope (SEM, Czech Republic FEI Co., Ltd., operating at $10 \mathrm{kV}$ ). The silver content of the dressings was determined by inductively coupled plasma mass spectrometry (ICP-MS, Agilent 7500ce, USA).

2.3.2 Asymmetric modification measurement. Ink was dripped onto the hydrophilic and hydrophobic surfaces of the CTS-SF/SA dressing at five different points, and photos of the droplets were recorded. The wettability of the CTS-SF/SA dressing was measured using a contact angle meter (Future
Digital Scientific, Asia Inc.) at room temperature. The contact angles were measured at each of the five different points on both the hydrophilic and hydrophobic surfaces.

2.3.3 Porosity measurement. The porosity of the prepared dressings was measured as described previously. ${ }^{27}$ The dressings were dipped into absolute ethanol. After being fully infiltrated for $2 \mathrm{~h}$ to obtain saturation, the dressings were immediately weighed. The porosity $(P)$ was calculated by the following equation:

$$
P=\frac{m_{2}-m_{1}}{\rho V} \times 100 \%
$$

in this equation, $m_{1}$ and $m_{2}$ are the weights of the dressing before and after soaking in absolute ethanol for $2 \mathrm{~h}$, respectively; $V$ is the volume of the dressing before immersion; and $\rho$ is the density of alcohol at $25{ }^{\circ} \mathrm{C}$, i.e., $0.785 \mathrm{~g} \mathrm{~cm}^{-3}$. All samples were tested in triplicate.

2.3.4 Degree of swelling and moisture-retention capacity. The degree of swelling (DS) of the dressings was measured as previously reported..$^{28}$ The dressing samples were dipped into normal saline until saturation. Then, the dressings were removed, their surfaces were scrubbed softly, and the samples were immediately weighed. The weights of the dressings were measured repeatedly until the weight was constant over three measurements to ensure the dressing had reached saturation. The weight of the samples was recorded before $\left(m_{0}\right)$ and after immersion in normal saline for a given time $\left(m_{\mathrm{w}}\right)$. The formula used to determine the DS (\%) of the developed dressings is provided below.

$$
\mathrm{DS}=\frac{m_{\mathrm{w}}-m_{0}}{m_{0}} \times 100 \%
$$

To measure the moisture-retention capacity of the dressing, the wet dressing was placed in a glass dryer at room temperature with the modified side up and the unmodified side down, and the DS was determined every $1.5 \mathrm{~h}$. All samples were tested in triplicate.

2.3.5 Evaluation of the mechanical properties. The tensile strength of the dressing (length $\times$ width $\times$ height: $50 \mathrm{~mm} \times$ $15 \mathrm{~mm} \times 2 \mathrm{~mm}$, dumbbell shape) was tested using a universal testing machine (SANS, CMT8202) with a crosshead speed of $20 \mathrm{~mm} \mathrm{~min}^{-1}$ at room temperature. Data were obtained from the average of five replicates for each sample.

\subsection{In vitro antibacterial test}

2.4.1 Testing the antibacterial and AgNP release properties of the dressings. The antibacterial activity of the dressing was tested via the agar diffusion method. ${ }^{29}$ S. aureus, E. coli, P. aeruginosa and $M$. albicans were used to evaluate the antibacterial activity of the dressing. First, $70 \mu \mathrm{l}$ of a bacterial suspension $(1 \times$


ilized dressings (CTS-SF/SA, CTS-SF Ag0.5/SA, CTS-SF Ag1.0/SA, and CTS-SF Ag2.0/SA, $\varnothing 1 \mathrm{~cm}$ ) were placed on the surface of the agar and incubated for $12 \mathrm{~h}$ at $37^{\circ} \mathrm{C}$. The experiment for each strain was repeated 3 times. Finally, the diameter of the bacteria-inhibiting ring was measured. 
To test the AgNP release properties of the dressings under simulated physiological conditions, we quantitatively measured the AgNP release of dressings with different amounts of AgNP in a solid medium. We added $1 \mathrm{ml}$ of agar medium in the liquid phase to a 24 well-plate, which was then cooled until it solidified at room temperature. Then, the sterilized dressings (CTS-SF Ag0.5/SA, CTS-SF Ag1.0/SA, and CTS-SF Ag2.0/SA, $\varnothing 1 \mathrm{~cm})$ were placed on the surface of the agar with the rough surface down for $24 \mathrm{~h}, 48 \mathrm{~h}$, and $72 \mathrm{~h}$ at $37^{\circ} \mathrm{C}$. The amount of silver on the agar blocks was then measured by ICP-MS.

2.4.2 Evaluation of the AgNP slow-release and microbe barrier properties. To evaluate the AgNP slow-release properties of the dressings, the antibacterial effects of the CTS-SF Ag0.5/SA dressing and a sample of gauze containing the same AgNP dose as the dressing (denoted Gauze-Ag dressing) were tested against $S$. aureus, E. coli, $P$. aeruginosa and $M$. albicans, with pristine gauze and the CTS-SF/SA dressing serving as controls. To ensure the Gauze-Ag dressing contained the same AgNP dose, $100 \mu \mathrm{l}$ of the AgNP solution $\left(18.25 \mu \mathrm{g} \mathrm{m}{ }^{-1}\right)$ was added to the gauze $(2 \mathrm{~cm} \times 2 \mathrm{~cm})$, which was then dried at room temperature.

To evaluate the resistance of the CTS-SF/SA dressing to the infiltration of microorganisms, $70 \mu \mathrm{l}$ of a $P$. aeruginosa suspension $\left(1 \times 10^{8} \mathrm{CFU} \mathrm{ml}{ }^{-1}\right)$ was dropped onto the smooth surface of the CTS-SF/SA and CTS-SF dressings, which were then placed on an LB agar plate with the modified side up and the unmodified side down, cultured for $24 \mathrm{~h}$ at $37{ }^{\circ} \mathrm{C}$ and recorded in detail with photos.

\subsection{Biosecurity evaluation}

The CTS-SF/SA, CTS-SF Ag0.5/SA, CTS-SF Ag1.0/SA and CTS-SF Ag2.0/SA (cobalt-60 sterilization processing) dressings were immersed in serum-free low-glucose DMEM (1 g sample in $100 \mathrm{ml}$ medium) and extracted for $24 \mathrm{~h}$ at $37^{\circ} \mathrm{C}$. Then, $10 \%$ FCS was added to the extract. The FCS/extract medium was used to culture L929 cells, human fibroblasts and HUCMSCs. The viability and proliferation of cells were determined by an MTT assay. The cells cultured in DMEM (containing 10\% FBS) were used as the control. All the experiments were performed in triplicate.

Standard tests for irritation and delayed-type hypersensitivity were performed using the aforementioned extract liquid in guinea pigs (wt: $200 \mathrm{~g}$ ) according to ISO 10993-10:2010: Biological Evaluation of Medical Devices.

\subsection{Evaluation of in vivo wound healing}

2.6.1 Establishment of the animal model. The experiments were conducted according to the current laws and the NIH guidelines for the Care and Use of Laboratory Animals (NIH Publications No. 80-23, revised 1996) and were approved by the Institutional Animal Care and Use Committee of the Academy of Military Medical Sciences Experimental Center (Beijing, China).

In total, $80 \mathrm{BALB} / \mathrm{c}$ male mice, 7-10 weeks old and weighing $18 \pm 2 \mathrm{~g}$, were randomly assigned into four groups with 20 mice in each group. The surgical procedures were performed under anaesthesia by intraperitoneally injecting pentobarbital sodium
(50 $\mathrm{mg} \mathrm{kg}^{-1}$ ). A full-thickness wound (diameter of $1 \mathrm{~cm}$ ) was created on the shaved dorsal side of each mouse. The wounds were inoculated with $P$. aeruginosa at $10^{8} \mathrm{CFU}(100 \mu \mathrm{l}$ per wound) for $20 \mathrm{~min}$ to obtain infected wound models. The wounds were then covered with $2 \mathrm{~cm} \times 2 \mathrm{~cm}$ samples of the CTS-SF Ag0.5/SA dressing, Gauze-Ag dressing, CTS-SF/SA dressing and plain gauze (modified side up and unmodified side down). The dressings were replaced every 2 days.

2.6.2 Wound healing rate. The wound areas were measured and photographed on the 4 th, 8 th, and 14 th day after operation. Images were quantified using the Image software (Imaging Processing and Analysis in Java, National Institutes of Health). The healing rate was calculated as the percentage of the healed area relative to the original wound size. ${ }^{30}$

2.6.3 Bacterial cultures of the exudate. On the 2 nd day after operation, wound exudates were collected using sterile swabs in a biosafety cabinet and cultured in LB broth for $4 \mathrm{~h}$ at $37^{\circ} \mathrm{C}$. Then, $75 \mu$ of the liquid was spread on agar medium and cultured for $8 \mathrm{~h}$ at $37^{\circ} \mathrm{C}$, and the colonies were observed.

2.6.4 Analysis of blood samples. A total of 80 blood samples were taken by removing mice eyeballs on the 2nd, 4 th, 8th, and 14th day after operation, and blood was collected in EDTA-K anticoagulation tubes. After gently oscillating the tubes up and down, the samples were injected into an automatic blood cell analyser (Sysmex CS-2000i Systems, Japan), and the white blood count (WBC), neutrophil granulocyte percentage (NEUT\%) and lymphocytes percentage (LYMPH\%) were analysed.

Standard blood samples were collected from another 5 BALB/c male mice (7-10 weeks old, $18 \pm 2 \mathrm{~g}$, from the same resource as the mice used in this experiment). These blood samples were analysed to determine the parameters of the blood samples prior to the operation.

2.6.5 Histological examination. Tissue samples were taken on the 8th and 14th day after operation and fixed in $10 \%$ formalin before fabricating paraffin sections. The samples were stained by the H\&E and Masson methods and observed using an optical microscope (DMI3000B, Leica). The silver content in the liver, spleen and kidney of the mice were determined through ICP-MS on the 14th day after the operation.

2.6.6 Statistics. All quantitative data were expressed as the mean with standard deviation (mean $\pm \mathrm{SD}$ ). Differences in the measured data were compared using the ANOVA method, and pairwise comparisons among the means were conducted by the LSD method. In this study, $p$-values less than 0.05 were considered statistically significant.

\section{Results and discussion}

\subsection{Characterization of AgNP and the CTS-SF Ag/SA dressings}

As illustrated in Fig. 2A and B, we prepared AgNP with good distribution. The particle size distribution of AgNP was mainly observed in about the main peak (50 nm) and few were distributed in the other peaks. The average value of main peak (peak 1) was $49 \mathrm{~nm}$. Inter-connective porous structures in the lyophilized sponge were observed by SEM. Although AgNP were 



Fig. 2 Characterization of AgNP and the CTS-SF Ag/SA dressings ((A) TEM images of AgNP; (B) nanoparticle size (diameter) distribution; (C) EDX spectrum of CTS-SF Ag0.5/SA dressing; (D1 and D2) SEM images of the CTS-SF/SA dressing surface; (E1 and E2) SEM images of the CTSSF Ag0.5/SA dressing surface; (F1 and F2) SEM images of the CTS-SF Ag1.0/SA dressing surface; (G1 and G2) SEM images of the CTS-SF Ag2.0/SA dressing surface).

added to the CTS-SF/SA dressing, they did not significantly affect the foaming emulsion due to the low dose of AgNP that was added. Therefore, the surface structure of the CTS-SF Ag0.5/ SA dressing was similar to that of the CTS-SF/SA dressing. With an increasing amount of AgNP, the surface pores of the CTS-SF Ag1.0/SA and CTS-SF Ag2.0/SA dressings were smaller and more uniform than those of the CTS-SF/SA dressing. As in shown in Fig. 2D-G, the number of particles in the pores of the dressing increased with an increasing AgNP content. This is in close agreement with the results of the silver contents in the dressings detected by ICP (Table 1).

\subsection{Analysis of the asymmetric modification of the dressing}

The good moisture retention of the dressing exhibited a positive effect on wound healing. Fig. 3A1 and A2 show the asymmetric wettability of the modified dressing (CTS-SF/SA). As shown in
Table 1 Silver content in the dressings

\begin{tabular}{ll}
\hline Materials & Silver content $\left(\mu \mathrm{g} \mathrm{g}^{-1}\right)$ \\
\hline CTS-SF & 0 \\
CTS-SF Ag0.5 & $9.7712 \pm 0.2266$ \\
CTS-SF Ag1.0 & $22.3911 \pm 0.1807$ \\
CTS-SF Ag2.0 & $47.9614 \pm 0.1687$
\end{tabular}

Fig. 3B1 and B2, ink drops were absorbed by the unmodified surface of the CTS-SF/SA dressing, but the modified surface exhibited hydrophobic characteristics. The asymmetric wettability of the CTS-SF/SA dressing was also evaluated by water contact angle measurements, with the unmodified surface exhibiting superhydrophilicity by immediately absorbing the water drops (Fig. 3C1), while the water contact angle of the modified hydrophobic upper surface was $105^{\circ}$, indicating high hydrophobicity (Fig. 3C2). The surface of the unmodified CTSSF/SA dressing was spongy and exhibited interconnected micropore structures (Fig. 3D1). The pore size distribution of hydrophilic surface was 100-400 $\mu \mathrm{m}$. The hydrophobic surface modified with stearic acid was smooth and have barely pores when comparing with hydrophilic surface (Fig. 3D1 and D2). The surface-modified CTS-SF/SA dressing had many advantages. The hydrophilic side could effectively absorb wound exudate and did not block the release of AgNP, allowing efficient antibacterial activity. Further, the side of the dressing that was exposed to the air was hydrophobic, which could greatly reduce the loss of water, thereby promoting wound healing. ${ }^{31,32}$ In addition, the hydrophobic layer could protect against microorganisms from the air, thus significantly reducing the risk of wound infection.

\subsection{Physical and mechanical properties}

Fig. 4A shows the porosities of the different dressings. All the dressings had porosities of $65-70 \%$, which indicates that the asymmetric wettability modification of the antibacterial dressing using SA did not significantly affect the porosity of the dressing itself. The pore volume of the wound dressing affects the rate of exudate uptake of the dressing. The high porosity of the dressing will promote the absorption of exudate from the wound to the infected necrotic tissue being removed. In addition, this high porosity can benefit the transmission of oxygen and nutrients into cells in the wound to promote wound healing. ${ }^{33}$

Fig. 4B shows the swelling ratio of the dressings under physiological conditions. After full immersion into NS, the swelling ratios of the CTS-SF and CTS-SF Ag dressings were in the range of 14-16, while those of the CTS-SF/SA and CTS-SF $\mathrm{Ag} / \mathrm{SA}$ dressings were in the range of 11-12, which were lower than that of the unmodified dressing. Fig. 4C shows that although the swelling ratio of the unmodified dressing (Fig. 4B) was higher than that of the modified dressings, the moisturepreserving time of the asymmetric-wettability-modified dressings was significantly higher than that of the unmodified dressing. The moisture-preserving times of the asymmetric- 
wettability-modified dressings were in the range of $16-17 \mathrm{~h}$, while the moisture-preserving time of the unmodified dressing was in the range of 10-12 h. Although the moisture level of the unmodified dressing was higher than that of the modified dressings, the water loss of the unmodified dressing was faster than that of the modified dressings with an increase in exposure time ( $>5.5 \mathrm{~h})$. After $5.5 \mathrm{~h}$, the moisture-preserving times of the modified dressings were higher than that of the unmodified dressing, and the final moisture-preserving times of the modified dressings were also longer than that of the unmodified dressing.

Because the asymmetric-wettability-modified dressings were placed with their hydrophobic surface facing up (simulated use of the modified dressings in wound nursing), effectively preventing the loss of internal moisture in the dressings, the moisture-preserving time of the modified dressings was extended. According to wound healing theory, a moist wound environment can promote wound healing and enable the painless removal of the dressing, thereby avoiding the destruction of fresh tissue and reducing scar formation. ${ }^{34-36}$


Fig. 3 Asymmetric modification measurement ((A1 and A2) schematic diagram for the modification of the dressing by stearic anhydride, where one side of the dressing is hydrophilic and the other side is hydrophobic; (B1) hydrophilic surface of the CTS-SF/SA dressing; (B2) hydrophobic surface of the CTS-SF/SA dressing; (C1) water contact angles of the hydrophilic surface; (C2) water contact angles of the hydrophobic surface); (D1) SEM images of hydrophilic surface of CTSSF/SA dressing; (D2) SEM images of hydrophobic surface of CTS-SF/SA dressing.
Fig. 4D shows the tensile strength measurements of the dressings. The tensile strength of the pristine CTS-SF dressing was 0.48 MPa. For the CTS-SF dressings with different AgNP concentrations, the tensile strengths of CTS-SF Ag0.5, CTS-SF Ag1.0 and CTS-SF Ag2.0 were reduced to $0.41,0.24$ and $0.13 \mathrm{MPa}$, respectively. The tensile strength decreased with an increase in the AgNP content, and this phenomenon was also noted in some previous reports. ${ }^{37,38}$ Although the tensile strengths of CTS-SF Ag were less than that of the CTS-SF dressing, these tensile strengths are suitable for wound care applications. Fig. 4D shows that the asymmetric wettability modification of the dressing had very little impact on the tensile strength.

\subsection{Antibacterial effect analysis}

Fig. 5A-D shows the antibacterial effect of the 4 types of dressings (CTS-SF/SA, CTS-SF Ag0.5/SA, CTS-SF Ag1.0/SA and CTS-SF Ag2.0/SA) on the 4 bacterial strains ( $P$. aeruginosa, $S$. aureus, $M$. albicans and E. coli). Fig. 5E visually demonstrates the diameter of the inhibition zone of the three kinds of CTS-SF $\mathrm{Ag} / \mathrm{SA}$ dressings against different strains. Compared with the effect on $P$. aeruginosa, $M$. albicans and $E$. coli, the antibacterial effect on $S$. aureus was poor. The lower antibacterial activity for $S$. aureus is most likely because $S$. aureus is Gram positive, and thus, its walls are composed of considerable amounts of peptidoglycan. Hence, the bacterial walls are thick and dense, while the walls of the other three strains, which are Gramnegative bacteria, are thin and loose and contain small amounts of peptidoglycan..$^{39,40}$ Note that although the AgNP contents of the CTS-SF Ag0.5/SA, CTS-SF Ag1.0/SA and CTS-SF Ag2.0/SA dressings were very different, an increase in the size of the inhibition rings was not obvious with an increase in the silver content.
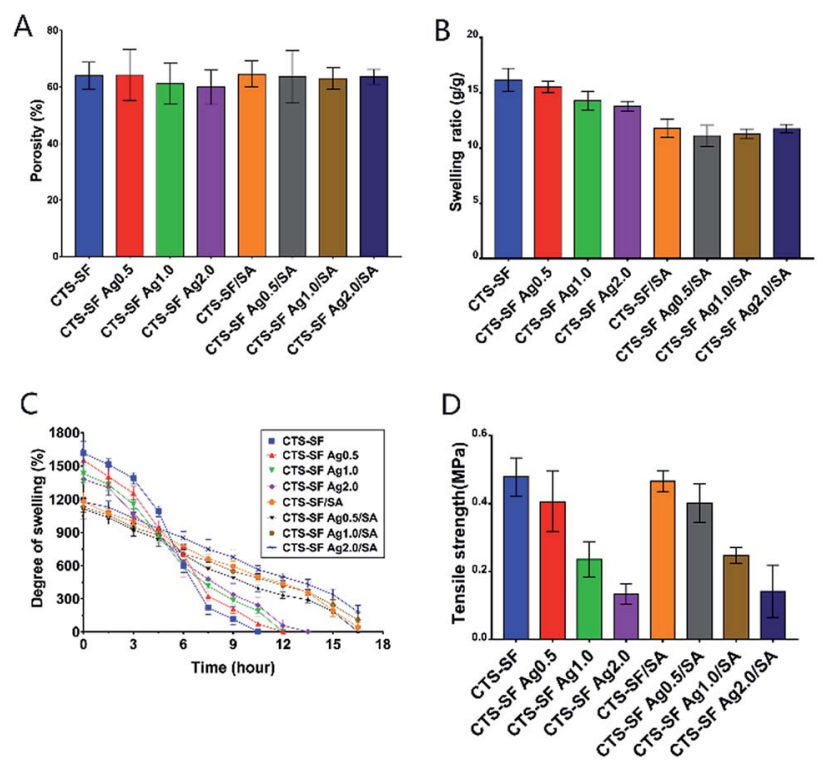

Fig. 4 Physical and mechanical properties of the dressings ((A) porosity; (B) swelling ratio; (C) moisture retention capacity; (D) tensile strength). 



0: CTS-SF/SA

2: CTS-SF Ag1.0/SA

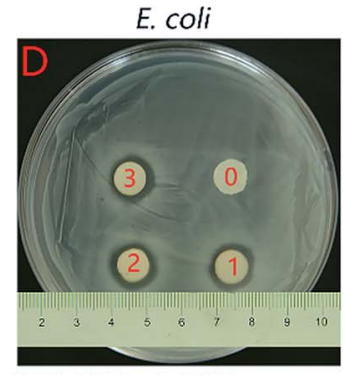

1: CTS-SF Ag0.5/SA

3: CTS-SF Ag2.0/SA

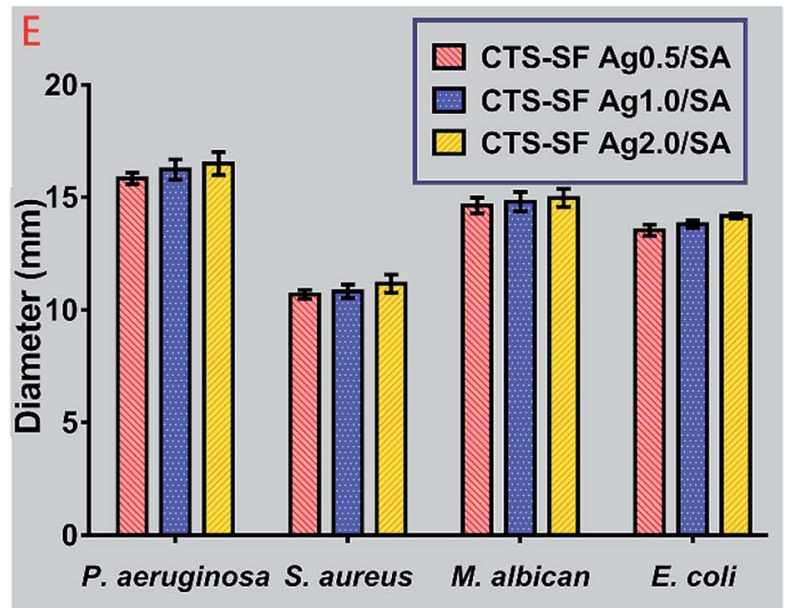

Fig. 5 The antibacterial effects of the different antimicrobial dressings on four bacterial strains ( $(\mathrm{A})$ the antibacterial effect on $P$. aeruginosa; (B) the antibacterial effect on S. aureus; (C) the antibacterial effect on $M$. albicans; (D) the antibacterial effect on E. coli; (E) diameter of the inhibition zone of dressings; 0: CTS-SF/SA dressing; (1) CTS-SF Ag0.5/SA dressing; (2) CTS-SF Ag1.0/SA dressing; (3) CTS-SF Ag2.0/SA dressing).

This finding is consistent with the results of the ICP test, given in Table 2. With an increase in the AgNP content in the dressings, the release of silver from the dressings in agar medium seems to increase. However, we found (as shown in ESI Table $1 \dagger$ ) no statistical difference in the release of silver from the CTS-SF Ag0.5/SA, CTS-SF Ag1.0/SA and CTS-SF Ag2.0/SA dressings $(p>0.05)$. This likely indicates that AgNP are trapped inside the pores of the dressings, and thus, they have a very slow release. Also, we compared the silver release from the same kind of dressing after submerging in agar medium for $24 \mathrm{~h}, 48 \mathrm{~h}$ and $72 \mathrm{~h}$ using the ANOVA method, as shown in ESI Table 2. $\dagger \mathrm{A}$ significant statistical difference was observed $(p<0.05)$. We found that as the time increased, the silver release continued to
Table 2 The amount of silver release in agar medium

\begin{tabular}{lllr}
\hline & \multicolumn{3}{l}{ The amount of silver release in agar medium $\left(\mu \mathrm{g} \mathrm{g}^{-1}\right)$} \\
\cline { 2 - 4 } Time $(\mathrm{h})$ & CTS-SF Ag0.5/SA & CTS-SF Ag1.0/SA & CTS-SF Ag2.0/SA \\
\hline $24 \mathrm{~h}$ & $0.0679 \pm 0.0132$ & $0.0684 \pm 0.0202$ & $0.0718 \pm 0.0196$ \\
$48 \mathrm{~h}$ & $0.0888 \pm 0.0032$ & $0.0904 \pm 0.0124$ & $0.094 \pm 0.0165$ \\
$72 \mathrm{~h}$ & $0.1019 \pm 0.0131$ & $0.1328 \pm 0.0107$ & $0.1423 \pm 0.0242$
\end{tabular}

increase for the CTS-SF Ag0.5/SA, CTS-SF Ag1.0/SA and CTS-SF Ag2.0/SA dressings. This finding also suggests that AgNP can undergo a slow and sustained release in combination with the CTS-SF/SA sponge scaffold.

\subsection{Evaluation of the slow release of AgNP and the microbe barrier}

Compared with the gauze sample loaded with the same AgNP dose (Gauze-Ag), CTS-SF Ag0.5/SA can slowly release AgNP into the wound, and its asymmetric modification was beneficial for protecting against bacteria from the external environment (as shown in Fig. 6). Fig. 6A-D shows the inhibition rings for $P$. aeruginosa, $S$. aureus, $M$. albicans and $E$. coli cultured with the CTS-SF Ag0.5/SA dressing and the Gauze-Ag dressing, which indicate the antibacterial effect of the CTS-SF Ag0.5/SA and Gauze-Ag dressings on the four strains.

The inhibition rings of the Gauze-Ag dressing were significantly larger than those of the CTS-SF Ag0.5/SA dressing, thus demonstrating that AgNP are suddenly released from the GauzeAg dressing. By contrast, the CTS-SFAg0.5/SA composite dressing not only has good antibacterial properties but also can slowly release AgNP into the wound. The P. aeruginosa solution was dropped onto the smooth surface of the CTS-SF and CTS-SF/SA dressings, and after being cultured for $24 \mathrm{~h}$ at $37^{\circ} \mathrm{C}$, obvious bacterial plaques were observed in the CTS-SF dressing group, but no bacterial plaque was found in the CTS-SF/SA dressing group, apart from on traces of the dressing (Fig. 6E-G). This finding illustrates that the asymmetrical modification of the CTS-SF/SA dressing can effectively protect the wound from microorganisms. When the CTS-SF/SA dressing covers a wound on the skin, the upper hydrophobic surface can effectively protect the wound from infection from bacteria in the air.

\subsection{Biosecurity test}

We also examined the biocompatibility and toxicity of the CTSSF/SA, CTS-SF Ag0.5/SA, CTS-SF Ag1.0/SA and CTS-SF Ag2.0/SA dressings both in vitro and in vivo, as shown in Fig. 7. Fibroblasts play a very important role in the wound healing process, ${ }^{\mathbf{4 1}}$ and the wound itself can induce stem cells (such as mesenchymal stem cells) to participate in wound repair. ${ }^{42}$ Therefore, we used L929 cells, human fibroblasts and HUCMSCs to test the cytocompatibility of the four types of dressings. The results in Fig. 7A-C show that the cell viability in the three cell types cultured with the CTS-SF/SA dressing extracts were higher than that in the controls, which suggests that low-molecular-weight silk fibroin and chitosan were dissolved in the extracts, and 

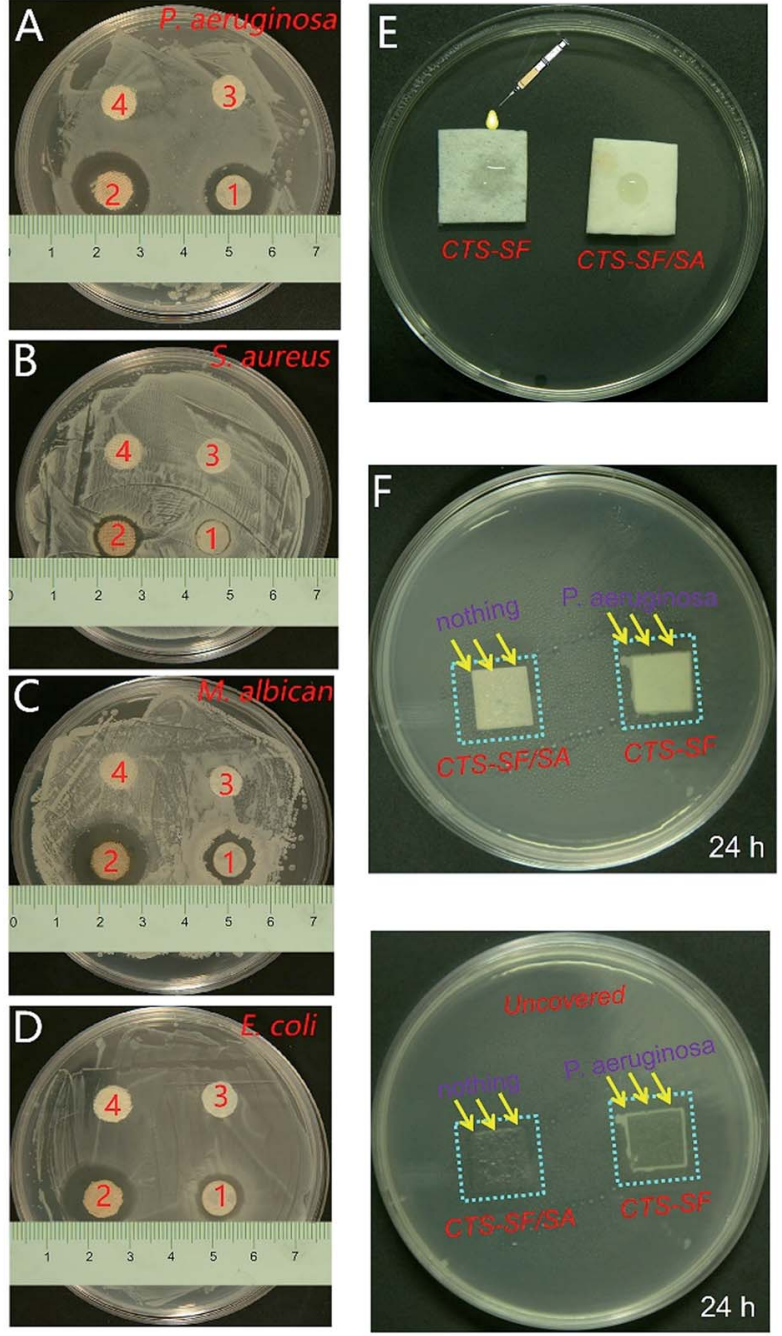
1: CTS-SF Ag0.5/SA
2: Gauze-Ag
3: CTS-SF/SA
4: Gauze

Fig. 6 Controlled AgNP release from the CTS-SF Ag0.5/SA dressing and its resistance to the infiltration of microorganisms ((A-D) 1, 2, 3 and 4 indicate the antibacterial effects on $P$. aeruginosa, $S$. aureus, $M$. albicans and $E$. coli, respectively; (E) operation chart of the experiment, where the hydrophilic surfaces of the CTS-SF/SA and CTS-SF dressings were in contact with the LB medium and a $P$. aeruginosa suspension was dropped on the upper surfaces of the CTS-SF/SA and CTS-SF dressings, respectively; (F) cultured for $24 \mathrm{~h}$ at $37{ }^{\circ} \mathrm{C}$; (G) observation of the uncovered dressing).

these two compounds (especially silk fibroin) played a positive role in cell proliferation. However, the viability of the three types of cells cultured with the CTS-SF Ag0.5/SA, CTS-SF Ag1.0/SA and CTS-SF Ag2.0/SA dressing extracts decreased with an increase in AgNP, but there was no difference between the CTS-SF Ag0.5/SA dressing extracts and the controls.

The results of the subcutaneous sensitization test in guinea pigs demonstrate mild allergic reactions in the CTS-SF Ag1.0/SA and CTS-SF Ag2.0/SA dressing groups at $24 \mathrm{~h}$ and $48 \mathrm{~h}$, but no allergic reactions were observed in the NS, CTS-SF/SA dressing and CTS-SF Ag0.5/SA dressing groups at $24 \mathrm{~h}$ and $48 \mathrm{~h}$. Finally,


Fig. 7 Biosecurity test ((A) cell biocompatibility test of the modified dressing extracts to L929 cell lines; (B) cell biocompatibility test of the modified dressing extracts to human fibroblasts; (C) cell biocompatibility test of the modified dressing extracts to HUCMSCs; (D) standard tests for irritation and delayed-type hypersensitivity).

based on the integration of the above antibacterial tests (Fig. 5A1-A3), we selected the CTS-SF Ag0.5/SA dressing for the treatment of infected wounds.

\subsection{In vivo evaluation of the effect on wound healing}

The effect of the CTS-SF Ag0.5/SA dressing on wound healing was evaluated by observing the infected wound repair in $\mathrm{BALB} / \mathrm{c}$ mice. Fig. 8 shows the healing of the infected wound with the CTS-SF Ag0.5/SA dressing, Gauze-Ag, CTS-SF/SA dressing and Gauze groups on the 4 th, 8 th and 14th day. In clinical practice, nano-silver antibacterial dressings are mostly composed of AgNP absorbed by gauze. To guarantee the scientific rationality of the measurement of the accumulated silver content in the organs of mice on the 14th day, we added the same amount of AgNP to gauze (i.e., the Gauze-Ag group) as that added to the CTS-SF Ag0.5/SA dressing to evaluate the controlled release of AgNP in both dressing groups. Observing the mice wound model on the 4th day, we found different levels of scabbing in the wound treated by the four dressings, and wound contraction had begun. The wound contraction levels with the CTS-SF Ag0.5/SA dressing, CTS-SF/SA dressing and Gauze groups were more conspicuous. As shown in Fig. 8E, the wound healing levels were quantitatively evaluated at different times. On the 4th day, the wound healing ratio in the CTS-SF Ag0.5/SA dressing group was $48.04 \pm 3.0 \%$, and the wound healing ratios in the CTS-SF/SA, Gauze-Ag and Gauze dressing groups were $40.68 \pm 2.0 \%, 22.84 \pm 2.2 \%$ and $34.08 \pm 2.4 \%$, respectively. No redness was observed at the edge of the wounds in the CTS-SF Ag0.5/SA or CTS-SF/SA dressing groups. However, obvious swelling was observed at the edge of wounds in the Gauze-Ag group, and some infected exudations were observed in the Gauze group. Observing the mice wound model on the 8th day, we found that the cuts in the CTS-SF Ag0.5/SA and CTS- 
$0 \mathrm{~d}$
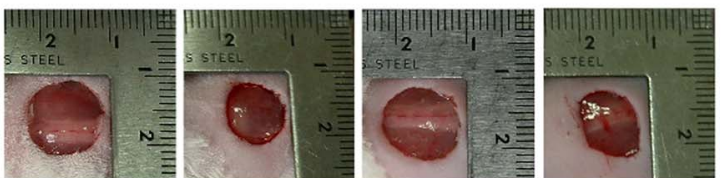

$4 d$
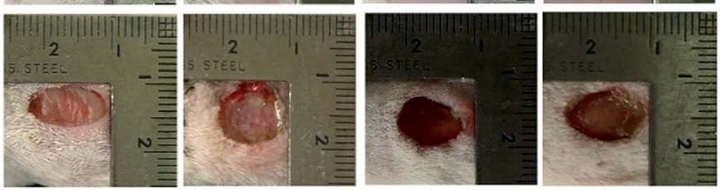

$8 d$


$14 \mathrm{~d}$



CTS-SF Ag0.5/SA

Gauze-Ag

CTS-SF/SA

Gauze

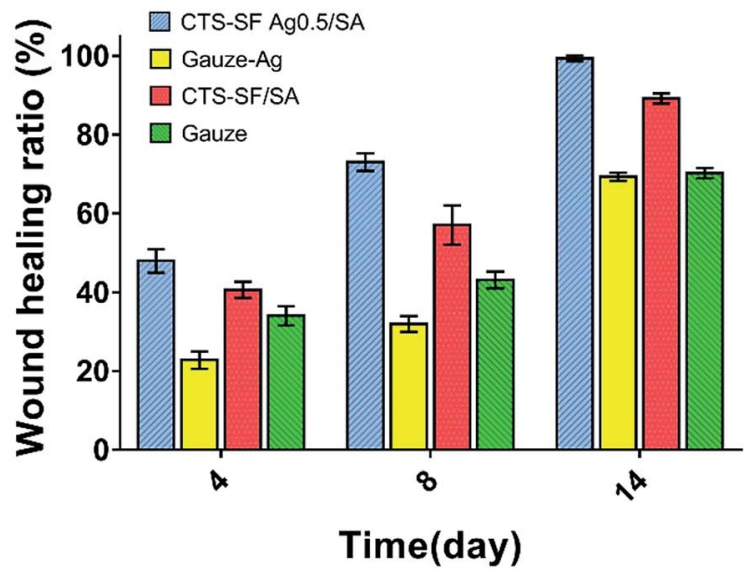

Fig. 8 Analysis of the healing ratio of an infected wound ((A-D) photographs of the wounds on the 0,4 th, 8th and 14th day; (A1-A4) group treated by the CTS-SF Ag0.5/SA dressing; (B1-B4) group treated by the Gauze-Ag dressing; (C1-C4) group treated by the CTS-SF/SA dressing; (D1-D4) group treated by gauze; $(E)$ wound healing ratio on the 4 th, 8 th and 14 th day).

SF/SA dressing groups had been sloughed away, and wound contraction in the CTS-SF Ag0.5/SA dressing group was the most obvious, exhibiting a healing rate of $73.05 \%$. Further, severe adhesion was found between the dressing and wound in the Gauze-Ag group, with the obvious appearance of redness on the edge of the wound, and considerable amounts of granulation tissue grew and some inflammatory exudates appeared in the Gauze group. On the 14th postoperative day, the wound healing ratio of the CTS-SF Ag0.5/SA group reached $99.38 \pm 1.5 \%$, while the healing ratios in the CTS-SF/SA dressing, Gauze-Ag and Gauze groups were $89.22 \pm 1.3 \%, 69.26 \pm 3.7 \%$ and $70.23 \pm$ $1.3 \%$, respectively. The four groups exhibited a significant difference in terms of the healing ratio on the 14th day (Table 3); namely, the CTS-SF Ag0.5/SA dressing group performed the best, followed by the CTS-SF/SA dressing group, with the Gauze-
$\mathrm{Ag}$ and Gauze groups performing the poorest. We also found that the renascent skin following treatment with the CTS-SF Ag0.5/SA and CTS-SF/SA dressings was smooth with no scarring, similar to normal skin. This indicates that the CTS-SF Ag0.5/SA dressing has a positive effect on the healing of skin tissue.

The results of the in vivo infected wound healing suggest that the CTS-SF Ag0.5/SA dressing did not stimulate tissue and could fight infection while accelerating wound healing, especially in the initial stages of healing. Continuous observations of the wound healing process indicated that the CTS-SF Ag0.5/SA dressing can more effectively play a role in fighting infection and maintaining a moist wound micro-environment to accelerate wound constriction and healing.

\subsection{Exudation cultivation and analysis of the immune response}

On the 2nd postoperative day, the mice were sacrificed, and the body surfaces were disinfected. In a biosafety cabinet, wound excretion was sampled with a sterile plastic rod and added into the LB fluid medium. After $4 \mathrm{~h}$, no turbidity was observed in the media of the CTS-SF Ag0.5/SA dressing and Gauze-Ag groups (Fig. 9A1 and A2), but the media became turbid in the CTS-SF/SA dressing and Gauze groups (Fig. 9A3 and A4). For further analysis, $50 \mu \mathrm{l}$ of LB fluid medium was extracted from each group and spread on an agar plate, and $8 \mathrm{~h}$ later no colony growth was apparent in the CTS-SF Ag0.5/SA dressing and Gauze-Ag groups. However, in the CTS-SF/SA dressing and Gauze groups, colonies had grown. The results show that the CTS-SF Ag0.5/SA dressing and Gauze-Ag can kill P. aeruginosa in the wound, but the CTS-SF/SA dressing and Gauze were not able to kill this bacterium.

In this study, we evaluated the immune response by analysing three types of blood parameters (i.e., WBC count, NEUT\% and $\mathrm{LYMPH} \%$ ) after trauma and infection (Fig. 9B-D) to evaluate the antibacterial effects of the four types of dressings. On the 2nd day after wound infection, no significant change in the three parameters was observed between the four groups because the host immune response was not fully activated during the early stage of infection. On the 4th day, in the CTSSF/SA and Gauze groups, the WBC count and NEUT\% showed a significant increasing trend, but LYMPH\% decreased significantly. However, on the same day, the WBC count, NEUT\% and LYMPH\% had not changed significantly in the CTS-SF Ag0.5/SA dressing and Gauze-Ag dressing groups. These results confirmed that the CTS-SF Ag0.5/SA dressing and Gauze-Ag dressing could effectively kill $P$. aeruginosa in the wound and decrease the immune response caused by infection. Fig. 9B-D show that on the 8th day, the WBC and NEUT\% continued to grow, and LYMPH\% decreased, which indicates that the peak of the immune response had been reached. Interestingly, the WBC count and NEUT\% surged on the 8th day in the Gauze group, which was mainly due to the delayed effect of wound healing. The high concentrations of AgNP used to treat the wound for a long duration may have led to this delay in healing, which caused the WBC count and NEUT\% to surge significantly. 
process of repair was inactive. In contrast, the CTS-SF Ag0.5/SA and CTS-SF/SA dressing groups exhibited better repair capacities. Considerable capillary vessels and abundant fibrocytes were observed in the CTS-SF Ag0.5/SA and CTS-SF/SA dressing groups, and the former exhibited very few inflammatory cells. This finding indicates that the asymmetric-wettability-modified chitosan dressing could improve the wound healing and vessel formation abilities. Meanwhile, low doses of AgNP could reduce the inflammatory response of the body. H\&E staining tissue biopsies on the 14th day (Fig. 10) showed that the tissue was actively repaired in the dermis layer, accompanied by an inflammatory reaction and erosion in parts of the epidermis in the Gauze-Ag dressing group, while the structure of the epidermis was coherent in the Gauze group. At the same time, the epidermis in the CTS-SF Ag0.5/SA and CTS-SF/SA dressing groups was completely repaired, with keratinization and a dermis that was rich with blood vessels. In addition, immature hair follicles and thick-walled vessels were observed in the CTS-SF Ag0.5/SA dressing group. The results of the tissue section on the 14th day demonstrated that the wound had finished repairing and remodelling in the CTS-SF Ag0.5/SA dressing group. From a microscopic perspective, the process of wound healing was conducted mainly by the formation of collagen and the proliferation of fibrocytes. Therefore, evaluating the collagen deposition during the process of wound healing is very important. The results of Masson staining (Fig. 10) were basically consistent with the H\&E staining results. On the 14th day, collagen deposition in the CTS-SF Ag0.5/SA and CTS-SF/SA dressing groups was more robust than that in the Gauze-Ag dressing and Gauze groups. Among these groups, the wound in the CTS-SF Ag0.5/SA dressing group showed the most significant collagen expression, and the tissue of the dermis was closer that of normal skin, with cells in the dermis layers arranged regularly.

In contrast to the CTS-SF Ag0.5/SA and CTS-SF/SA dressing groups, the Gauze-Ag and Gauze groups significantly lacked collagen. The collagenous fibres of the dermis tissues were arranged loosely and disorderly in the Gauze-Ag dressing group. The results of the above histologic analysis demonstrate that the CTS-SF Ag0.5/SA dressing is a good antibacterial repair material. This dressing can accelerate the healing process and quickly restore the normal structure and function of skin tissue.

Table 4 shows the silver content in the organs of mice treated with the CTS-SF Ag0.5/SA dressing, Gauze-Ag dressing, CTS-SF/ SA dressing and Gauze on the 14th day. No silver was detected in the Gauze or CTS-SF/SA dressing groups, but silver was widely

Table 4 Silver content in the organs of mice on the 14th day

\begin{tabular}{llll}
\hline & Organs of mice & \\
\cline { 2 - 4 } Dressing & Liver $\left(\mu \mathrm{g} \mathrm{g}^{-1}\right)$ & Spleen $\left(\mu \mathrm{g} \mathrm{g}^{-1}\right)$ & Kidney $\left(\mu \mathrm{g} \mathrm{g}^{-1}\right)$ \\
\hline CTS-SF Ag0.5/SA & $0.0105 \pm 0.0012$ & $0.0387 \pm 0.0012$ & $0.0516 \pm 0.0030$ \\
Gauze-Ag & $0.1587 \pm 0.0034$ & $0.0415 \pm 0.0028$ & $0.0509 \pm 0.0018$ \\
CTS-SF/SA & 0 & 0 & 0 \\
Gauze & 0 & 0 & 0
\end{tabular}

detected in the liver, spleen and kidney after treatment with the Gauze-Ag dressing, and the highest amount of silver was found in the liver of mice $\left(0.157 \pm 0.0034 \mathrm{mg} \mathrm{kg}^{-1}\right)$. However, the amount of silver in the liver of mice treated with the CTS-SF Ag0.5/SA dressing $\left(0.01046 \pm 0.0012 \mathrm{mg} \mathrm{kg}^{-1}\right)$ was $1 / 15$ of that in mice treated with the Gauze-Ag dressing $(0.157 \pm$ $0.0034 \mathrm{mg} \mathrm{kg}^{-1}$ ). The good AgNP slow-release property of the CTS-SF Ag0.5/SA dressing demonstrates its good biosecurity and biocompatibility.

\section{Conclusions}

In conclusion, we designed a CTS-SF Ag/SA dressing that was asymmetrically modified with SA and could slowly release AgNP. The prepared CTS-SF Ag0.5/SA dressing had good structural channels, hydroscopicity and moisture retention, as well as adequate mechanical integrity. More importantly, in addition to exhibiting antibacterial functionality, the dressing also promoted healing. The dressing performed well in tests of physical performance, antimicrobial properties, infiltration resistance towards microorganisms, biosecurity and in the treatment of infected wounds. This study is expected to provide a solution for the repair of infected wounds and will hopefully provide a scientific basis for the development of related biomedical treatments.

\section{Conflicts of interest}

There are no conflicts to declare.

\section{Acknowledgements}

This work was supported by the National Natural Science Foundation of China (81541111, 31470938, 31170926, 21134004, 11421202, 61227902, and 11120101001), International Joint Research Center of Aerospace Biotechnology and Medical Engineering from Ministry of Science and Technology of China, 111 Project (B13003), Research Fund for the Doctoral Program of Higher Education of China (20131102130004), The transformation project for major achievements of Central Universities in Beijing (ZDZH20141000601), and Fundamental Research Funds for the Central Universities.

\section{References}

1 E. A. Ayello and S. Baranoski, Nursing, 2014, 27, 380-382.

2 A. V. Rawlings and C. R. Harding, Moisturization and skin barrier function, 2004.

3 A. Summerfield, F. Meurens and M. E. Ricklin, Mol. Immunol., 2014, 66, 14-21.

4 M. C. Robson, Surg. Clin. North Am., 1997, 77, 637.

5 A. Griffiths-Jones, J. Wound. Care., 1995, 4, 481-483.

6 P. Deichmann, A. Sura, C. Sanders, N. Aravindakshan-Patel and F. Lopez, J. La. State Med. Soc., 2017, 169, 20.

7 X. H. Niu and X. L. Li, Zhonghua Shaoshang Zazhi, 2016, 32, 71-73. 
8 J. Chai, Z. Sheng and J. Gao, Chin. Crit. Care Med., 1999, 12, 721-724.

9 S. S. Bleehen, D. J. Gould, C. I. Harrington, T. E. Durrant, D. N. Slater and J. C. Underwood, Br. J. Dermatol., 1981, 104, 19.

10 J. J. Hostýnek, R. S. Hinz, C. R. Lorence, M. Price and R. H. Guy, Crit. Rev. Toxicol., 1993, 23, 171-235.

11 J. Chen, C. M. Han and C. H. Yu, Chin. J. Burns, 2004, 20, 161. 12 X. W. Wang, N. Z. Wang, O. Z. Zhang, R. L. Zapata-Sirvent and J. W. Davies, Burns Incl. Therm. Inj., 1985, 11, 197-201.

13 S. Sano, R. Fujimori, M. Takashima and Y. Itokawa, Burns Incl. Therm. Inj., 1982, 8, 278-285.

14 M. G. Boosalis, J. T. Mccall, D. H. Ahrenholz, L. D. Solem and C. J. Mcclain, Surgery, 1987, 101, 40-43.

15 F. Han, Y. Dong, Z. Su, R. Yin, A. Song and S. Li, Int. J. Pharm., 2014, 476, 124-133.

16 L. N. Ke, X. M. Feng and C. R. Wang, J. Clin. Rehabil. Tissue Eng. Res., 2010, 14, 521-524.

17 D. Liang, L. Zhong, Y. Hao, J. Gao and C. Rong, ACS Appl. Mater. Interfaces, 2016, 8, 3958.

18 Y. Liu, J. H. Xin and C. H. Choi, Langmuir, 2016, 28, 17426.

19 N. A. Ivanova and A. B. Philipchenko, Appl. Surf. Sci., 2012, 263, 783-787.

20 J. P. Chen, G. Y. Chang and J. K. Chen, Colloids Surf., A, 2008, 313-314, 183-188.

21 P. T. Kumar, V. K. Lakshmanan, T. V. Anilkumar, C. Ramya, P. Reshmi, A. G. Unnikrishnan, S. V. Nair and R. Jayakumar, ACS Appl. Mater. Interfaces, 2012, 4, 2618.

22 E. Zakhem, S. Raghavan, R. R. Gilmont and K. N. Bitar, Biomaterials, 2012, 33, 4810.

23 M. A. Brown, M. R. Daya and J. A. Worley, J. Emerg. Med., 2009, 37, 1-7.

24 A. Mandal, S. Sekar, N. Chandrasekaran, A. Mukherjee and T. P. Sastry, J. Mater. Chem. B, 2015, 3, 3032-3043.

25 N. Duraipandy, R. Lakra, K. V. Srivatsan, U. Ramamoorthy, P. S. Korrapati and M. S. Kiran, J. Mater. Chem. B, 2015, 3, 1415-1425.

26 D. Liang, Z. Lu, H. Yang, J. Gao and R. Chen, ACS Appl. Mater. Interfaces, 2016, 8, 3958.
27 P. T. S. Kumar, S. Abhilash, K. Manzoor, S. V. Nair, H. Tamura and R. Jayakumar, Carbohydr. Polym., 2010, 80, 761-767.

28 G. Ramanathan, S. Singaravelu, M. D. Raja, N. Nagiah, P. Padmapriya, K. Ruban, K. Kaveri, T. S. Natarajan, U. T. Sivagnanam and P. T. Perumal, $R S C A d v ., 2016,6$, 7914-7922.

29 J. W. Rhim, S. I. Hong, H. M. Park and P. K. Ng, J. Agric. Food Chem., 2006, 54, 5814-5822.

30 S. M. Bauer, L. J. Goldstein, R. J. Bauer, H. Chen, M. Putt and O. C. Velazquez, J. Vasc. Surg., 2006, 43, 134-141.

31 S. Metzger, Home Healthc. Nurse, 2004, 22, 586.

32 R. White, MA Healthcare, London, 2003, vol. 8(suppl. 5), p. S3.

33 A. Mata, E. J. Kim, C. A. Boehm, A. J. Fleischman, G. F. Muschler and S. Roy, Biomaterials, 2009, 30, 4610-4617. 34 G. D. Winter, Nature, 1963, 200, 378-379.

35 D. Okan, K. Woo, E. A. Ayello and G. Sibbald, Adv. Skin Wound Care, 2007, 20, 53-55.

36 D. A. Burgos, J. Giménez, E. Moreno, E. Lamberto, M. Utrera, E. M. Urraca, F. J. Vélez, E. López, M. A. Martínez and M. J. Gómez, Clin. Drug Invest., 2000, 19, 357-365.

37 S. Shankar and J.-W. Rhim, Carbohydr. Polym., 2015, 130, 353-363.

38 N. Eghbalifam, M. Frounchi and S. Dadbin, Int. J. Biol. Macromol., 2015, 80, 170-176.

39 J. J. Antony, P. Sivalingam, D. Siva, S. Kamalakkannan, K. Anbarasu, R. Sukirtha, M. Krishnan and S. Achiraman, Colloids Surf., B, 2011, 88, 134-140.

40 A. M. Fayaz, K. Balaji, M. Girilal, R. Yadav and R. Venketesan, Nanomedicine, 2009, 6, 103-109.

41 H. Bouissou, M. T. Pieraggi and J. C. Thiers, J. Soc. Biol., 1999, 193, 41-48.

42 D. H. Hu and W. Zhang, Zhonghua Shaoshang Zazhi, 2017, 33, 9.

43 S. B. Bai, G. D. Wang and W. U. Yang, Prog. Mod. Biomed., 2011, 17, 3370.

44 Y. S. Wu and S. N. Chen, Front. Pharmacol., 2014, 5, 1. 45 V. Falanga, Lancet, 2005, 366, 1736. 